\title{
Exaggerated Waiting Impulsivity Associated with Human Binge Drinking, and High Alcohol Consumption in Mice
}

\author{
Sandra Sanchez-Roige', Victor Baro', Leanne Trick', Yolanda Peña-Oliver', David N Stephens' and \\ Theodora Duka', \\ 'School of Psychology, University of Sussex, Falmer, Brighton, UK
}

\begin{abstract}
There are well-established links between impulsivity and alcohol use in humans and animal models; however, whether exaggerated impulsivity is a premorbid risk factor or a consequence of alcohol intake remains unclear. In a first approach, human young (I 8-25 years) social binge and non-binge drinkers were tested for motor impulsivity and attentional abilities in a human version of the Five-Choice Serial Reaction Time Task (Sx-5CSRTT), modeled on the rodent 5CSRTT. Participants completed four variants of the Sx-5CSRT, in addition to being screened for impulsive traits (BIS-I I questionnaire) and impulsive behavior (by means of the Delay Discounting Questionnaire, Two-Choice Impulsivity Paradigm (TCIP), Stop Signal Reaction Time, and Time Estimation Task). Using a second approach, we compared one of these impulsivity measures, 5CSRTT performance, in two inbred strains of mice known to differ in alcohol intake. Compared with non-bingers (NBD; $n=22)$, binge drinkers $(B D, n=22)$ showed robust impairments in attention and premature responding when evaluated under increased attentional load, in addition to presenting deficits in decision making using the TCIP. The best predictors for high binge drinking score were premature responding in the Sx-5CSRTT, trait impulsivity in the BIS-I I, and decision making in the TCIP. Alcohol-naïve C57BL/6J (B6) mice (alcohol preferring) were more impulsive in the 5CSRTT than DBA2/J (D2) mice (alcohol averse); the degree of impulsivity correlated with subsequent alcohol consumption. Homologous measures in animal and human studies indicate increased premature responding in young social BD and in the ethanol-preferring B6 strain of mice.
\end{abstract}

Neuropsychopharmacology (2014) 39, 29|9-2927; doi: |0.1038/npp.2014.15 I; published online 16 July 2014

Impulsivity has been consistently implicated as both a determinant and a consequence of alcohol abuse. Binge patterns of alcohol consumption, in particular, have been associated with impaired attentional function and executive function (Scaife and Duka, 2009; Townshend and Duka, 2005), and increased impulsive behavior in both humans and rodent models (Bell et al, 2013; Duka et al, 2003; Stephens and Duka, 2008). However, it is unclear whether high levels of impulsivity are a cause or consequence of alcohol intake.

Given the prevalence of binge drinking in adolescence (Healey et al, 2014), the first aim of our study was to examine the relationship of binge drinking to measures of waiting impulsivity and attentional abilities in human adolescents (18-25 years old) and in rodents, which consume alcohol. Taking the rodent 5CSRTT (Robbins, 2002) as a model, we developed a novel, iPad-based task (Sussex 5CSRTT; Sx-5CSRTT), to assess both attentional performance and anticipatory behavior in humans. BD were also characterized in four additional measures of impulsivity,

*Correspondence: Dr T Duka, School of Psychology, University of Sussex, Falmer, Brighton BNI 9QG, UK, Tel: + I 44 I 273 678879, Fax: +44 1273 678058, E-mail: t.duka@sussex.ac.uk

Received 28 March 20 I4; revised 9 June 20 I4; accepted II June 20 I4; accepted article preview online 20 June 2014 based on different operational definitions of the construct. The Stop Signal Task, used to assess ability to inhibit a prepotent response (Logan, 1994), served as an additional measure of 'motoric impulsivity'. The Delay Discounting Questionnaire measured preference for immediate overdelayed rewards (Petry, 2001) and, combined with the Two-Choice Impulsivity paradigm (Dougherty et al, 2005), provided an index of 'choice' impulsivity or decision making; the fourth behavioral measure used was the Time Estimation Task. Although each of these five measures has been used in the clinical context as an index of impulsive behavior, the sensitivity of each task to characterize impulsive phenotype in young social BD, and their relationship to one another has not been explored. We anticipated binge drinking might be differentially associated with different aspects of impulsive behavior, but generally that high binge drinking scores would be associated with high levels of trait and behavioral motor impulsivity and aversion to delay.

The role of premorbid impulsivity as a predictor of elevated alcohol intake cannot be easily disentangled in human studies, as impulsivity measures are almost inevitably assessed after a period of alcohol use. Animal models are more powerful tools in this respect as they allow the exclusion of alcohol experience as a potential contributor to impulsivity. Therefore, having established that 5CSRTT waiting impulsivity was associated with human 
binge drinking, we asked whether waiting impulsivity in alcohol-naïve mice predicted alcohol drinking in two widely used B6 and D2 inbred strains, that also differ in alcohol consumption (Crabbe et al, 1994). We have previously reported (Walker et al, 2011) that prior exposure to high alcohol concentrations over several weeks in adult hood has only transitory effects in increasing impulsivity in B6 mice. We predicted greater impulsivity in the highethanol-preferring mice indicating a potential causal relationship between waiting impulsivity and high alcohol consumption.

Using homologous measures of impulsivity in mouse and humans, we provide evidence that waiting impulsivity is associated with binge drinking in young adult humans, and predicts alcohol consumption in mice.

\section{MATERIALS AND METHODS}

\section{Human Study}

Recruitment and procedure. In total, 44 participants (22 male; age $18-25$ years, $M=21.18, S D=1.89$ ), recruited from the University of Sussex subject pool, were assigned to the binge (binge score $>32 ; \mathrm{BD}$ ) or non-binge (binge score $<16$; NBD) condition using scores from Alcohol Use Questionnaire (Mehrabian and Russell, 1978). To assess alcohol drinking patterns, a 'binge drinking' score (Townshend and Duka, 2002), was calculated based on the speed of drinking (number of drinks per hour), the number of episodes of alcohol intoxication in the past 6 months, and the percentage of alcohol intoxications out of the total number of times of going out drinking (see Supplementary Material). An overall score for weekly alcohol-unit consumption was also estimated.

Participants were healthy social drinkers (see Supplementary Material for further details of inclusion criteria). Upon arrival at the laboratory, a breathalyser (Lion Alcolmeter SD-400; Lion Laboratories, Barry, UK) was used to ensure zero breath alcohol levels. Participants completed: (a) the Barratt impulsivity scale, version 11 (Patton et al, 1995), a 30-item checklist that gives a total impulsivity score and three sub-scores of attentional, motor, and nonplanning impulsiveness; (b) the Alcohol Use Disorder Identification Test (AUDIT) (Saunders et al, 1993), to evaluate heavy drinking and/or active alcohol abuse or dependence; and (c) the National Adult Reading Task (NART) (Nelson and O'Connell, 1978), an estimate of verbal IQ. Following instructions and practice trials, participants were presented with five computerized tasks in random order (see below). At the end of the session $(90 \mathrm{~min}$ ), subjects were debriefed, informed of risks associated with binge drinking, and entered into a prize draw to win $£ 25$. All participants gave informed consent to take part in the study, which was approved by the University of Sussex ethics committee.

Behavioral measures of impulsivity. The Sx-5CSRTT was administered using an iPad (iOS 6 operating system; Apple), programmed in Mac OS X (Apple). Figure 1 depicts an example trial of the task. In brief, participants were required to detect and respond to the brief $(0.5 \mathrm{~s})$ highlighting of one of five moving visual stimuli. Responding before stimulus onset was considered a measure of poor inhibitory control, recorded as a premature response and followed by a 5-s time-out period. Following practice trials in which the stimulus was presented every $5 \mathrm{~s}$ (ITI 5-s), participants performed four task variants: a fixed (fITI) and a variable (vITI) session under simple task conditions; and, in order to increase the attentional load, a fITI and vITI session in combination with a dual task (Hogarth et al, 2008) in which subjects were also required to respond to a $659 \mathrm{~Hz}$ tone by performing a key press with the non-dominant hand. Main outcome variables were 'accuracy', 'percentage of omissions', and 'percentage of premature responding'.

The Stop Signal task (SST) (Logan, 1994) to test response inhibition; a delay discounting questionnaire (DDQ) (Petry, 2001) and Two-Choice Impulsivity paradigm (TCIP) (Dougherty et al, 2005), to assess preference for a small immediate over a large delayed reward, and the Time Estimation Task (TE) to evaluate the subject's time perception were added. Main outcome variables included the Go RT and calculated SSRTi from SST; the slope and area of the discounting curve ( $k$ and AUC parameters) from DDQ, and proportion of immediate choices and maximum number of consecutive delayed choices from the TCIP; and the subject's accuracy of performance in TE. See Supplementary Material for details of the tasks and analysis of main variables.

\section{Mouse Study}

Subjects. Two cohorts of mice from B6 $(n=21)$ and D2 $(n=22)$ strains, purchased from The Jackson Laboratory (Bar Harbor, Maine, USA), were used. Mice were housed in groups of two per cage on a 12-h light/dark cycle (lights off at 1900 hours) at a temperature of $19-21^{\circ} \mathrm{C}$ and $50 \%$ humidity. Before starting 5CSRTT training, mice were food restricted to reduce their body weights to $85 \%$ of their freefeeding weight. Water was available ad libitum. Behavioral testing took place between 0800 and 1400 hours, 5-6 days per week. Experiments were approved by the institutional ethics committee and performed under United Kingdom legislation on animal experimentation (Animal (Scientific Procedures) Act, 1986).

5CSRTT. Testing of performance followed the protocol previously described (Pena-Oliver et al, 2012; see Supplementary Methods). In brief, following training under fITI conditions, mice were tested under vITI $(2,5,10,15 \mathrm{~s})$ conditions to increase premature responding (Robbins, 2002; Sanchez-Roige et al, 2012).

Alcohol consumption. Following completion of the 5CSRTT, mice were given free access to food and water for 14 days before being tested for alcohol consumption and preference, using a two-bottle choice test (Belknap et al, 1993). See Supplementary Material for details.

Statistical analysis. Statistical analysis was performed using the 'Statistical Package for Social Sciences' (SPSS, version 20.0). Following two-way ANOVA with group (two levels: $\mathrm{BD}, \mathrm{NBD}$ ) and gender (two levels) as between subject factors, group differences were explored using one-way 
a
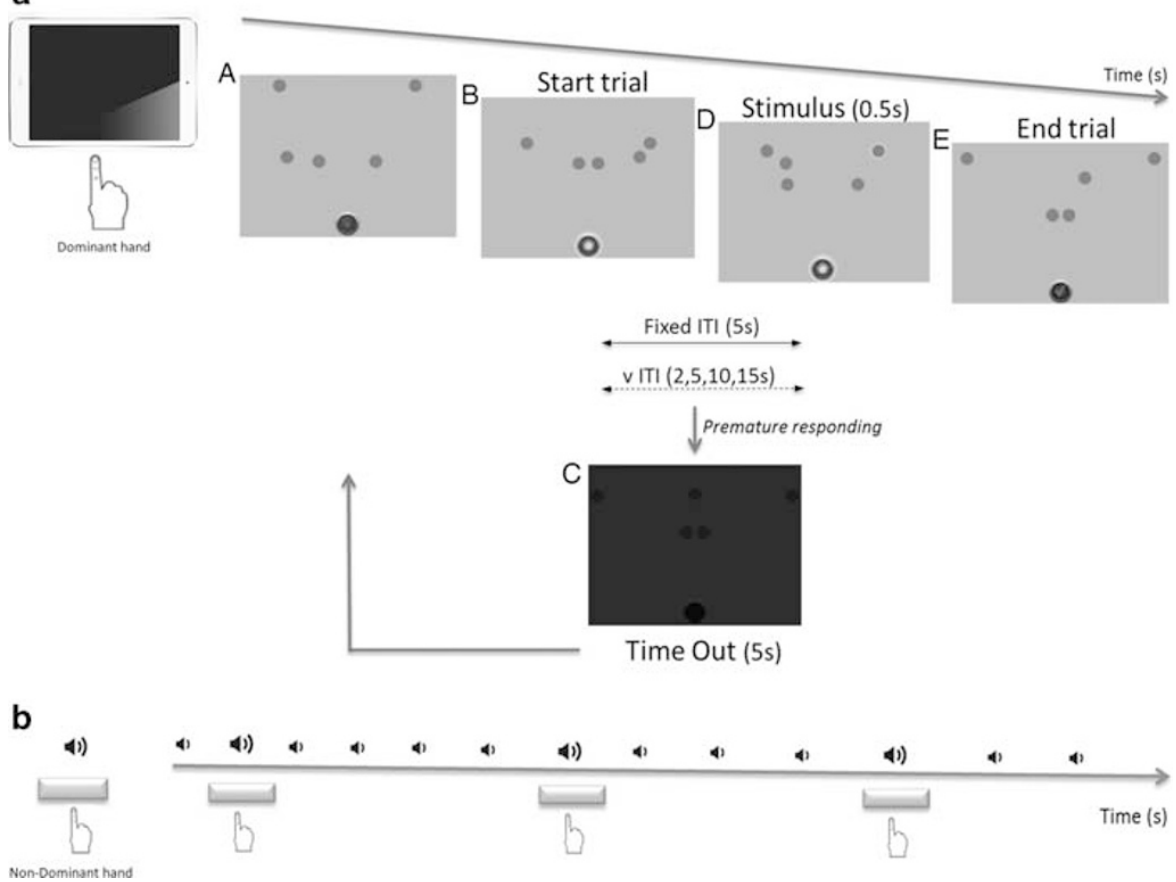

Figure I (A) Participants were comfortably seated in front of a touch screen. Viewing distance was approximately $30 \mathrm{~cm}$ with a vertical visual angle of $-30^{\circ}$ and a horizontal visual angle of $0^{\circ}$. The task consisted of five independently moving blue circles (stimulus) represented in a 'circular' motion in a tactile screen. We adopted moving targets in an attempt to increase attentional load, which, in the mouse task, comes about because reinforcer retrieval, as well as spontaneous locomotor activity diverts attention from the five-choice array. Below the stimuli and at the bottom of the screen, a home button was located. Trial commenced by the illumination of the house button (B). The participant was required to tap and hold onto the home button, and withhold responding until the stimulus presentation. After a designated inter-trial interval (ITl; s), one of the five circular visual stimuli modified its contour (D) and the participant was then required to tap into the highlighted circle and return to the home button. Illumination of the home button signaled the start of a new trial (E). Omissions (failure to respond to the signaled stimulus within a concrete period of time), incorrect responses (tapping into a non-designated circle), and premature responses (responses into the circles during the inter-trial interval before the stimulus presentation) were followed by a designated time-out period of $5 \mathrm{~s}(\mathrm{C})$. Perseverative responses (responding repeatedly to the circles after a correct detection) were also assessed. Total number of trials completed was determined, providing a measure of motivation. Following practice trials (correctly responding in each of the five signaled stimuli, or after 3 min, whichever came first), participants performed four task variants: a fITI and vITI session under simple task conditions ( $n=3 \mathrm{I}-32$; panel a); and a fITI and VITI session in combination with a dual task $(n=44$; panel b). During the dual task, participants were required to discriminate between sequences of lowand high-pitched tones ( 13 blocks of 10 trials; I high tone/block, presented in random order) and respond to the latter by pressing a space bar located in an external keyboard whilst performing the Sx-5CSRTT with their dominant index finger.

ANOVA. 'Binge drinking' scores were square-root transformed to obtain homogeneity of variance, though untransformed means are shown throughout. Daily cigarette use, total BIS score, the behavioral measures of 'accuracy' (fITI and vITI with dual task), 'percentage of omissions' (fITI and vITI with dual task), and 'percentage of prematures' (vITI with dual task) were analyzed by Mann-Whitney $U$-tests.

Pearson's correlation coefficient $r$ was used to determine relationships between Binge Scores and behavioral impulsivity measures and trait impulsivity measures from BIS-11. Trait impulsivity from BIS-11 was also correlated with the impulsivity measures. Finally, the variables 'SSRT', 'maximum delayed choices', 'BIS-trait', and 'premature responding' (the main variables denoting impulsivity) were entered into multiple regression analyses with binge drinking score as the dependent variable. Outliers ( $>3$ SD above group mean) were removed. Assumptions of normality (ShapiroWilk statistic) and homogeneity of variance (Levene's test) were met, unless otherwise stated.

Repeated measures ANOVA was used to compare performance across ITI conditions (four levels) as within-subject factors and strain as between factors. Where sphericity assumptions were violated, the Greenhouse-Geisser correction was applied and epsilon $(\varepsilon)$ values reported.

\section{RESULTS}

\section{Human Study}

In total, $22 \mathrm{BD}$ were compared with $22 \mathrm{NBD}$. Groups were matched on gender and IQ, but BD were younger $(F(1,43)=14.712, p=0.001)$, reported an earlier drinking onset $(F(1,42)=4.707, p=0.036)$ and presented higher scores on the AUDIT Test $(F(1,43)=23.214, p=0.001)$. Compared with NBD, BD subjects presented higher binge drinking scores (see Table 1; $F(1,43)=296.443, p=0.001$ ). $\mathrm{BD}$ subjects displayed significantly higher scores in the motor impulsivity and non-planning subscales of the BIS-11 questionnaire $(F(1,43)=6.820, p=0.012 ; F(1,43)=4.525$, $p=0.039$, respectively; Supplementary Figure S1), and a marginal tendency to present higher total scores of trait impulsivity $(U(42)=164.5, p=0.068)$. There were no 
Table I Group Characteristics (Age, Smoking Per Day, Verbal IQ, Alcohol use, Onset of Drinking) and Additional Impulsivity Measures for $\mathrm{NBD}$ and $\mathrm{BD}$

\begin{tabular}{|c|c|c|c|}
\hline Variable & Non-binge drinkers & Binge drinkers & Statistics \\
\hline N & $22(|| M, \mid I F)$ & $22(|| M, \mid I F)$ & \\
\hline$A g e^{a}$ & $22.14(1.83)$ & $20.23(1.44)$ & $F(1,43)=|4.7| 2, p=0.00 \mid$ \\
\hline Binge drinking score $¥^{a}$ & $5.96(4.08)$ & $48.39(13.10)$ & $F(I, 43)=296.443, p=0.001$ \\
\hline AUQ_weekly units ${ }^{\mathrm{a}, \mathrm{b}}$ & $10.10(8.34)$ & $25.22(13.23)$ & $F(1,43)=23.618, p=0.001$ \\
\hline AUDIT $^{\mathrm{a}}$ & $5.77(3.85)$ & $12.59(5.40)$ & $F(1,43)=23.210, p=0.001$ \\
\hline \multicolumn{4}{|l|}{ Barratt impulsivity scale } \\
\hline Total score $\beta$ & $61.50(10.34)$ & $68.90(7.61)$ & $U(42)=164.5, p=0.068$ \\
\hline \multicolumn{4}{|l|}{ Stop signal task } \\
\hline Go reaction time & $448.57(28.81)$ & $423.26(28.09)$ & $F(1,43)=0.270, p=0.606$ \\
\hline SSRTi & $169.65(12.52)$ & | $87.67(\mid 2.00)$ & $F(1,43)=0.899, p=0.348$ \\
\hline \multicolumn{4}{|l|}{ Delay discounting } \\
\hline$A \cup C$ & $0.27(0.27)$ & $0.23(0.26)$ & $F(1,43)=0.169, p=0.683$ \\
\hline
\end{tabular}

Abbreviations: AUQ, Alcohol Use Questionnaire; AUC, area under the curve of delay discounting; NART, National Adult Reading Test; verbal IQ; SSRTi, stop signal reaction times (milliseconds, $\mathrm{ms}$ ).

Values are expressed as mean \pm SD. Delayed choices = maximum number of consecutive delayed choices in a Two-Choice paradigm.

$\beta$, non-parametric; $¥$, SQRT transformed.

${ }^{a}$ Significant group differences.

${ }^{\mathrm{b}}$ One alcohol unit $=8 \mathrm{~g}$ of alcohol.

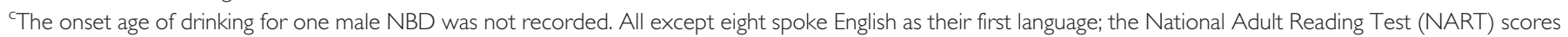
from these eight were discarded.

statistical group differences on scores for the attentional subscale $(F(1,43)=2.344, p=0.133)$.

Binge Drinkers' Performance on the Sussex-Five-Choice Serial Reaction Time Task

Simple task conditions. During the fITI session, BD displayed higher levels of premature responding $(F(1,30)=$ 4.656, $p=0.039$; Figure 2c) compared with NBD, but a group $\times$ gender interaction indicated that male BD showed more premature responding than male $\operatorname{NBD}(F(1,27)=$ $4.655, p=0.04$; Supplementary Figure S3A). No other group differences were found $(F<3, p>0.05)$.

During the vITI session, no group differences on premature responding or attentional (accuracy and omissions) performance were found $(F<1.6, p>0.05)$. A significant group $\times$ gender interaction revealed lower levels of accuracy in male $\mathrm{BD}$ than male $\mathrm{NBD}(F(1,28)=6.058, p=0.02$; Supplementary Figure S3A).

Dual-task conditions. During the fITI dual-task session, group differences appeared in measures of attention; BD showed lower accuracy $(U(44)=157, \quad p=0.027$;
Figure 2d) and higher levels of omissions $(U(44)=138$, $p=0.019$; Figure 2e). No effects of binge drinking were detected for premature responding ( $p>0.05$; Figure $2 \mathrm{f}$ ).

When participants performed a vITI dual-task session, attentional deficits in $\mathrm{BD}$ were again detected; $\mathrm{BD}$ subjects showed lower accuracy $(U(44)=146.5, p=0.009$; Figure $2 \mathrm{~d})$ and more omissions $(U(44)=161, p=0.049$; Figure 2e). Under these task conditions, BD also showed high percentage of premature responses $(U(44)=118, p=0.003$; Figure 2f).

The groups did not significantly differ on accuracy in detecting tones during the dual task in any of the conditions $(F<3.8, p>0.05)$.

\section{Binge Drinkers' Performance on Additional Behavioral Measures of Impulsivity}

There were no group differences in any of the SST measures $(F<1.4, p>0.05)$. Similarly, with regards to DDQ 'choice' impulsivity, both groups showed a similar linear decrease of indifference point as a function of increased delay $\left(R^{2}\right.$ values ranged from 0.92 to $0.94 ; k$ values for NBD and BD were 0.008 and 0.01 , respectively; see Table 1 ). In contrast, $\mathrm{BD}$ chose 


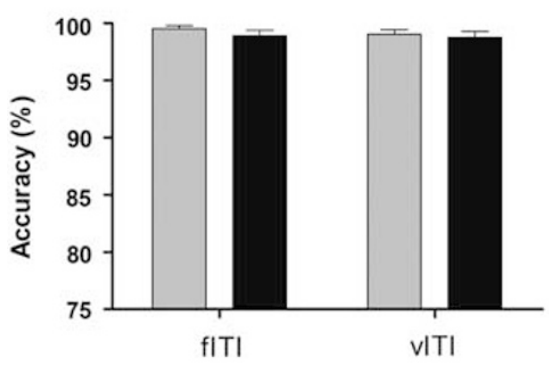

d

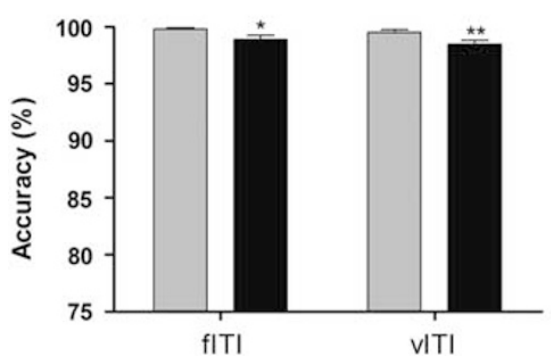

b

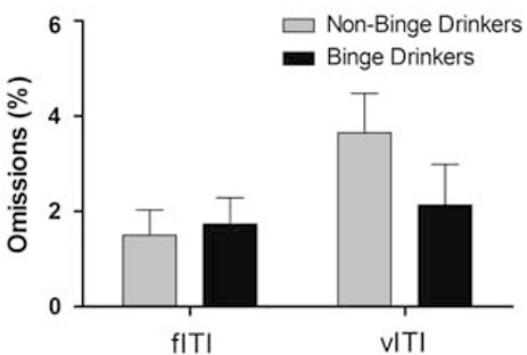

e

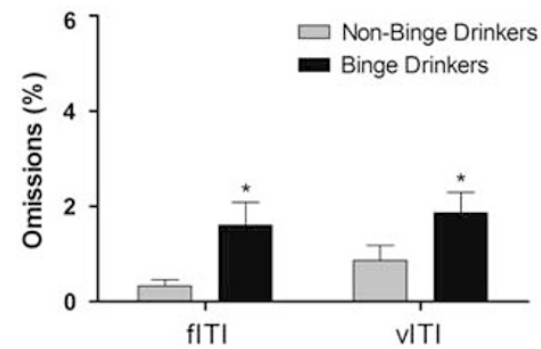

C

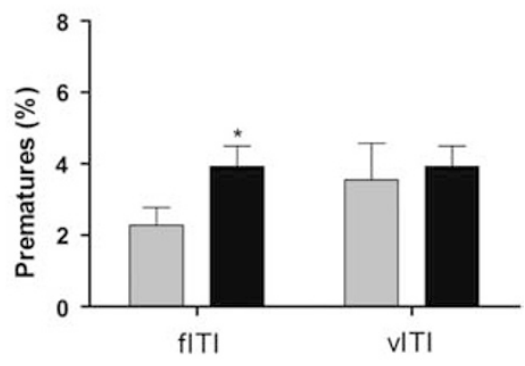

f

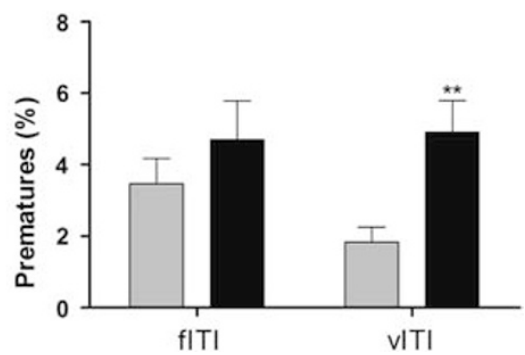

Figure 2 Sx-5CSRTT performance during a fixed ITI (fITI) and vITI sessions under simple task (top panels) and dual-task (bottom panels) conditions for NBD and BD. Mean ( \pm SEM) of the percentage of ( $a, d)$ accuracy of responding, (b, e) percentage of omissions, and (c, f) premature responses. * $p<0.05$, *** $p<0.0$ I compared with NBD (independent sample $t$-test or non-parametric Mann-Whitney U-test).
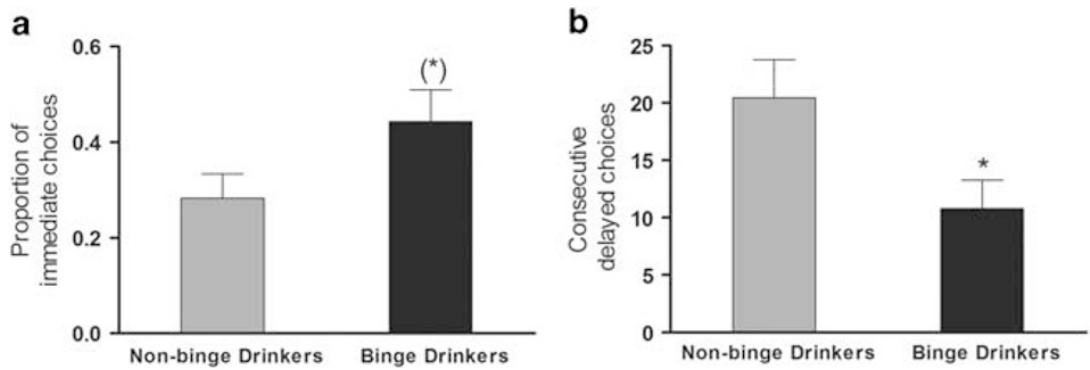

Figure 3 Performance during the Two-Choice Impulsivity paradigm. Mean \pm SEM of (a) proportion of immediate choices and (b) maximum number of consecutive delayed choices. ${ }^{*} p<0.05$ compared with NBD (independent sample t-test), (*) $p=0.064$.

delayed options less frequently than NBD during the TCIP $(F(1,43)=5.533, p=0.023$; Figure $3 b)$ and a tendency to display more immediate choices was detected $(F(1,43)=3.627$, $p=0.064$; Figure 3a). One-way ANOVA failed to reveal group differences on the accuracy of time estimation $(F(1,43)=1.31$, $p=0.259)$.

Gender differences in performance were detected only during the SST; female subjects displayed faster Go RT than males $(F(1,43)=4.407, p=0.042)$. There were no group $\times$ gender interactions in any tasks $(F<2.7, p>0.05)$.

Correlations between binge drinking scores and impulsivity measures. Significant positive correlations were found between binge scores and levels of BIS motor impulsivity trait $(r(44)=0.38, p=0.011)$ and behavioral measures of Sx-5CSRTT premature responding during fITI under simple task conditions $(r(30)=0.413, p=0.021)$ and during vITI under dual-task conditions $(r(31)=0.370, p=0.013)$.
Significant correlations were also detected between binge drinking scores and attentional measures in the Sx5CSRTT under dual-task conditions (percentage of omissions during vITI $(r(31)=0.363, p=0.015)$ and accuracy of responding during both fITI $(r(31)=-0.342, p=0.026)$ and vITI $(r(32)=-0.362, p=0.016)$; higher binge scores associated with higher omission rates and lower accuracy, respectively.

High binge drinking scores correlated with lower numbers of consecutive delay choices (TCIP; $r(44)=-0.392, p=0.029)$. No other planned correlations showed significance $(p>0.05)$.

In the multiple regression analysis, the main model was significant $(F(3,30)=7.314, p<0.01)$, and accounted for approximately $45 \%$ of the variance of binge drinking scores $\left(R^{2}=0.456, R^{2}\right.$ adjusted $\left.=0.396\right)$. Of the four factors included (SSRTi, maximum delayed choices, BIS-trait, and premature responding), only trait impulsivity $(\beta=0.454$, $p=0.004$ ) and premature responding during the first 

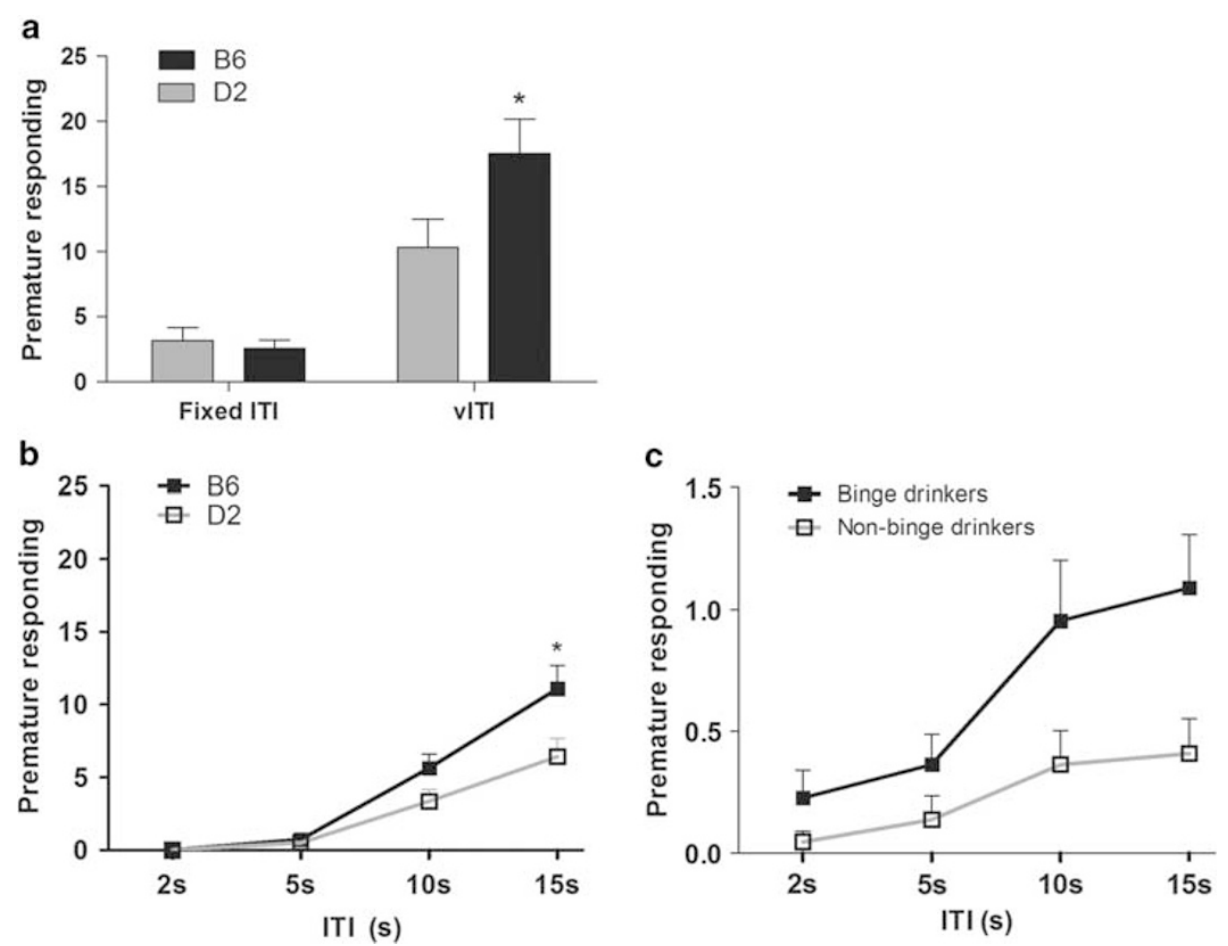

Figure 4 Premature responding (mean \pm SEM) of C57BL/6) (B6, black bars/lines) and DBA2/J (D2, gray bars/lines) in the 5CSRTT during a (a) flTI (last session in stage 6) and vITI sessions, and across the different ITIs during the vITI session for mice (b) and human participants (c). * $p<0.05$ (independent t-test).

(fITI-simple task; $\beta=0.369, p=0.016$ ) and last (vITIdual task; $\beta=0.324, p=0.033$ ) sessions were significant predictors of high binge drinking score $\left(R^{2}=0.46\right.$, $F(3,30)=7.55, p=0.001)$, with SSRTi and Delayed Choices not significantly contributing to the model $(\beta=-0.121$, $p=0.421 ; \beta=-0.250, p=0.086$, respectively).

\section{Mouse Study}

Strain differences in 5CSRTT impulsivity. As depicted in Figure $4 \mathrm{a}$, no differences in impulsivity were found between B6 and D2 mice during baseline conditions $(F(1,42)=0.248$, $p=0.621)$. Upon the introduction of vITI, the number of anticipatory responses in both strains increased $(F(3,123)=$ 68.457, $p=0.001, \varepsilon=0.415$ ); inspection across the different intervals during vITI revealed B6 mice as showing a steeper increase in premature responding with increasing ITI $($ ITI $\times$ strain interaction, $F(3,42)=4.930, p=0.024$; Figure $4 \mathrm{~b})$. Similarities across the increase in premature responding curves between mice and human subjects can be detected in Figure $4 \mathrm{~b}$ and $\mathrm{c}$.

Alcohol consumption. Alcohol consumption and preference for B6 and D2 mice is illustrated in Supplementary Figure S5. B6 consumed higher amounts of ethanol in comparison with D2 mice $(F(1,13)=37.244, p<0.001$; Supplementary Figure S5A) and showed greater ethanol preference ( $v s$ water, $F(1,13)=50.501, p<0.001$; Supplementary Figure S5B). Significant positive correlations were found between ethanol preference and number of premature responses during a vITI challenge (Spearman's rho(14) $=0.585, \quad p=0.028$; Supplementary Figure S5D); greater ethanol preference was associated with higher levels of premature responding in the 5CSRTT. Interestingly, significant correlations appeared at the longest ITI (15s, Spearman's rho(14) $=0.559, p=0.038)$, but only a tendency was revealed at 10s-ITI (Spearman's rho $(14)=0.518$, $p=0.058)$, with no significant correlations at 5s- or 2s-ITI (Spearman's rho $(14)<0.445, p>0.05)$. Levels of alcohol consumption did not correlate with levels of premature responding (Spearman's $\operatorname{rho}(14)=0.285, \quad p=0.324$; Supplementary Figure S5C).

\section{DISCUSSION}

Failure to control alcohol drinking has frequently been ascribed to 'impulsivity' (Dick et al, 2010), but this term is used to describe several distinct phenomena (Evenden, 1999). Furthermore, the extent to which 'impulsivity' is a cause or consequence of excessive alcohol consumption is not clear. Using a battery of tasks, we reveal impulsivity deficits in human BD, as indexed by the novel Sx-5CSRTT, and increased risky choices, measured in the TCIP. In parallel, we show alcohol-preferring B6 mice to be more impulsive in the mouse version of the 5CSRTT in comparison with alcohol-avoiding D2, and that waiting impulsivity correlates with alcohol preference. Thus, waiting impulsivity may precede heavy alcohol intake in the mouse, and, by extension, humans.

\section{Binge Drinking and Human 5CSRTT Performance}

Human BD showed elevated premature responding during the first (novelty component) and last (most challenging condition) session. Increased premature responding in BD 
subjects was accompanied by attentional impairments, revealed as more omitted trials, and lower accuracy of detection in the dual-task condition. Others have recently independently developed a similar human analog of the 5CSRTT to measure waiting impulsivity (Voon et al, 2014; Worbe et al, 2014), and have found parallels with rodent performance in substance abusers, and following neurochemical manipulations, suggesting close parallels between measures of waiting impulsivity in humans and rodents.

In our experiments, mouse performance in the 5CSRTT differed between B6 and D2 strains. Despite not differing during baseline performance (in keeping with reports from Loos et al, 2010; Patel et al, 2006), the ethanol-preferring B6 strain displayed higher levels of impulsivity when tested under challenging conditions by increasing the ITI, as did the humans (impaired performance was seen in the initial stages of the task and under attentional load).

Comparison of the mouse and human studies reveals that different manipulations of the task parameters lead to parallel behavioral changes. While a fITI session is often unable to detect group differences, increasing the time required to wait before responding, or making stimulus onset less predictable using a vITI, or increasing the task complexity, reveal attentional and impulsivity impairments in both our mouse and human studies. In vITI, the stimulus is presented using different inter-trial delays, in a semirandom fashion, within a single session, thus preventing the use of internal timing abilities to predict onset of the stimulus. Since impulsive individuals may overestimate the passage of time (Melges and Fougerousse, 1966), premature responses under fixed interval conditions may reflect errors in time estimation, rather than impulsivity. By disrupting the temporal predictability of the stimulus onset, the participant is required to be more attentive to the stimulus in order to achieve high levels of performance.

From the vITI, we can extract two main points; first, although vITI is sufficient to provoke premature responding in mice, vITI under the conditions described here was insufficient to detect differences in impulsivity between human groups, perhaps because in humans this variation is insufficient to challenge cognitive resources necessary for task performance. It was only when the complexity of the task was further extended by compromising attentional resources during dual-task performance that reliable group differences emerged. Second, from the vITI we can also observe a similarity in outcome between animal and human performance; the longer the ITI, the greater the challenge to the ability of the mouse or human to withhold responding. Interestingly, differences between $\mathrm{BD}$ and NBD (with $\mathrm{BD}$ showing more premature responses) were also detected during fITI in the simple task, when attention load was not challenged; however, these differences were found only when the task was performed for first time, before performance was optimized.

\section{Binge Drinking and Other Measures of Impulsivity}

Although increased 'waiting' impulsivity was detected in $\mathrm{BD}$, no differences in action inhibition or response cancelation appeared when using the SST, again emphasizing the multifaceted nature of impulsivity (Evenden, 1999).
Furthermore, the two tests that we used to cover other major impulsivity constructs, namely choice impulsivity or decision making, revealed increased impulsive choice in $\mathrm{BD}$ only in a behavioral task (TCIP), an effect consistent with previous reports of elevated risky choice in $\mathrm{BD}$ (Worbe et al, 2013), but not when using a questionnaire (DDQ), consistent with a number of researchers' findings when using the DDQ as a measure of impulsivity (Fernie et al, 2010; MacKillop et al, 2007). Data from correlational analysis of the human measures revealed positive relationships between percentage of premature responses during fITI and vITI, both behavioral measures of motor impulsivity, but self-reports were poorly correlated with behavioral task measures, consistent with previous studies highlighting robust differences between self-reported (Patton et al, 1995) and behavioral measures of impulsivity (Aichert et al, 2012; Dick et al, 2010; Vonmoos et al, 2013). Further, that measures of both impulsive choice (TCIP) and waiting impulsivity (Sx-5CSRTT) correlated with binge drinking score, but did not correlate with each other, might suggest that both forms of impulsivity contribute independently to poor control over alcohol consumption. It is therefore of interest that although B6 and D2 mouse strains also differ in measures of 'choice' impulsivity in delay-discounting tasks, surprisingly, greater discounting (higher impulsivity) has been reported in D2 than in B6 mice (Helms et al, 2006). Thus, whether the TCIP is a true measure of delay discounting, there is a mismatch between alcohol bingeing humans and alcohol-preferring mice.

It should be noted, however, that, in the TCIP, subjects choose between one stimulus that allows an immediate subsequent response for a small reward, and another that requires delaying the subsequent response to obtain a larger reward. Thus, the task not only opposes reward size to delay in obtaining it (as in standard delay-discounting tasks), but also incorporates a choice between responding quickly or following a delay. The task may thus contain an element of waiting impulsivity in addition to delay discounting.

In contrast to apparent species differences in delay discounting, BD humans and alcohol-preferring mice both showed high levels of waiting impulsivity in the 5CSRTT, suggesting homology between the tasks. Accepting this homology, the current data become important in interpreting whether changes seen in human $\mathrm{BD}$ precede or are the effect of binge drinking. That waiting impulsivity is seen in B6 mice that had not been exposed to alcohol drinking but are prone to alcohol abuse, and also in $\mathrm{BD}$, suggesting that high waiting impulsivity seen in BD subjects may not only be a consequence, but also may precede binge drinking. Although, only prospective studies in adolescents could finally identify behavioral predictors of alcohol binge drinking, studies like this one can guide us to include appropriate tasks in prospective studies.

\section{Limitations}

The development of homologous tasks in humans and animals will hopefully facilitate the translation of animal laboratory findings to human studies and, in a second step, to clinical populations. In that spirit, it is worth considering limitations of the study. First, although age of first drinking 
was provided by the participants, we have no independent measure of drinking patterns; such information would only be available from a prospective study. Similarly, illicit substance use was not formally assessed. Although corrections for multiple comparisons were not applied to the correlational data, the size effect of relationships between the important variables can still be used for interpretation of the findings. However, we recognize that our human findings should be considered exploratory and that impulsivity may account for only a small, albeit significant, proportion of differences in patterns of binge drinking. Despite gender-specific effects of binge drinking being reported frequently in the literature (Petry et al, 2002; Scaife and Duka, 2009), both male and female BD subjects were equally impaired when the demands of the task increased. The finding of increased premature responses in $\mathrm{BD}$ compared with NBD, in the simple form of the task and in the fITI condition, only in males is difficult to understand. This absence of clear gender differences may derive from our low sample size. Finally, although the present experiments suggest an association between high levels of waiting impulsivity and high alcohol consumption, we have no direct evidence of a causal relationship. It cannot be excluded, for instance, that gene variants that contribute to impulsivity, independently contribute to alcohol drinking (pleiotropy). Similarly, the significant correlations between impulsivity and alcohol consumption in the mouse experiments are only suggestive, and may be influenced by strain effects (see Supplementary Figure S5). Tests of a causal relationship need to demonstrate at least that manipulations of impulsivity have predictable consequences for drinking. The introduction of parallel tests in rodents and humans will facilitate such studies.

\section{CONCLUSIONS}

Altogether, the present findings suggest opportunities for obtaining consilience between animal and human studies of impulsivity. The Sx-5CSRTT was able to detect waiting impulsive elevations and attentional disruptions in a young human population of $\mathrm{BD}$, in line with previous studies describing alcohol-dependent patients with greater deficits in tasks related to prefrontal function (Scaife and Duka, 2009). Findings from our mouse study reveal that strains that differ in alcohol intake also show high levels of premature responding before alcohol exposure, and that the measure of impulsivity correlates with alcohol preference. Thus, trait impulsivity may predispose to binge drinking, as much as being a consequence of alcohol intake.

\section{FUNDING AND DISCLOSURE}

This work was supported by the European Commission InterReg project 'AlcoBinge'. The AlcoBinge project was selected within the context of the European programme of crossborder cooperation Interreg IV A France (Channel)England, co financed by ERDF. TD and DNS received research support from the UK Medical Research Council (grant G100008) and DNS from Glaxo SmithKline for research unrelated to the current report. The remaining authors declare no conflict of interest.

\section{ACKNOWLEDGEMENTS}

We thank Dr Kyriaki Nikolaou and Ms Sasha Ellis-Pridgeon for help in performing the human experiments.

\section{REFERENCES}

Aichert DS, Wostmann NM, Costa A, Macare C, Wenig JR, Moller HJ et al (2012). Associations between trait impulsivity and prepotent response inhibition. J Clin Exp Neuropsychol 34: 1016-1032.

Belknap JK, Crabbe JC, Young ER (1993). Voluntary consumption of ethanol in 15 inbred mouse strains. Psychopharmacology (Berl) 112: 503-510.

Bell RL, Franklin KM, Hauser SR, Engleman EA (2013). Next stop dependence. Binge drinking on the road to alcoholism: preclinical findings on its neurobiology from rat animal models. Binge Eating and Binge Drinking: Psychological, Social and Medical Implications. Nova Science Publishers: New York, pp 1-60.

Crabbe JC, Belknap JK, Buck KJ (1994). Genetic animal models of alcohol and drug abuse. Science 264: 1715-1723.

Dick DM, Smith G, Olausson P, Mitchell SH, Leeman RF, O’Malley SS et al (2010). Understanding the construct of impulsivity and its relationship to alcohol use disorders. Addict Biol 15: 217-226.

Dougherty DM, Mathias CW, Marsh DM, Jagar AA (2005). Laboratory behavioral measures of impulsivity. Behav Res Methods 37: 82-90.

Duka T, Townshend JM, Collier K, Stephens DN (2003). Impairment in cognitive functions after multiple detoxifications in alcoholic inpatients. Alcohol Clin Exp Res 27: 1563-1572.

Evenden JL (1999). Varieties of impulsivity. Psychopharmacology (Berl) 146: 348-361.

Fernie G, Cole JC, Goudie AJ, Field M (2010). Risk-taking but not response inhibition or delay discounting predict alcohol consumption in social drinkers. Drug Alcohol Depend 112: 54-61.

Healey C, Rahman A, Faizal M, Kinderman P (2014). Underage drinking in the UK: changing trends, impact and interventions. A rapid evidence synthesis. Int J Drug Policy 25: 124-132.

Helms CM, Reeves JM, Mitchell SH (2006). Impact of strain and D-amphetamine on impulsivity (delay discounting) in inbred mice. Psychopharmacology (Berl) 188: 144-151.

Hogarth L, Dickinson A, Janowski M, Nikitina A, Duka T (2008). The role of attentional bias in mediating human drug-seeking behaviour. Psychopharmacology (Berl) 201: 29-41.

Logan GD (1994). On the ability to inhibit thought and action: a users' guide to the stop signal paradigm. Inhibitory Processes in Attention, Memory, and Language In: Dagenbach D, Carr T H (eds) Academicpp 189-239.

Loos M, Staal J, Schoffelmeer AN, Smit AB, Spijker S, Pattij T et al (2010). Inhibitory control and response latency differences between C57BL/6J and DBA/2J mice in a Go/No-Go and 5choice serial reaction time task and strain-specific responsivity to amphetamine. Behav Brain Res 214: 216-224.

MacKillop J, Mattson RE, Anderson Mackillop EJ, Castelda BA, Donovick PJ (2007). Multidimensional assessment of impulsivity in undergraduate hazardous drinkers and controls. J Stud Alcohol Drugs 68: 785-788.

Mehrabian A, Russell JA (1978). A questionnaire measure of habitual alcohol use. Psychol Rep 43(3 Pt 1): 803-806.

Melges FT, Fougerousse CE Jr (1966). Time sense, emotions, and acute mental illness. J Psychiatr Res 4: 127-139.

Nelson HE, O'Connell A (1978). Dementia: the estimation of premorbid intelligence levels using the New Adult Reading Test. Cortex 14: 234-244. 
Patel S, Stolerman IP, Asherson P, Sluyter F (2006). Attentional performance of $\mathrm{C} 57 \mathrm{BL} / 6$ and DBA/2 mice in the 5-choice serial reaction time task. Behav Brain Res 170: 197-203.

Patton JH, Stanford MS, Barratt ES (1995). Factor structure of the Barratt Impulsiveness Scale. J Clin Psychol 51: 768-774.

Pena-Oliver Y, Buchman VL, Dalley JW, Robbins TW, Schumann G, Ripley TL et al (2012). Deletion of alpha-synuclein decreases impulsivity in mice. Genes Brain Behav 11: 137-146.

Petry NM (2001). Delay discounting of money and alcohol in actively using alcoholics, currently abstinent alcoholics, and controls. Psychopharmacology (Berl) 154: 243-250.

Petry NM, Kirby KN, Kranzler HR (2002). Effects of gender and family history of alcohol dependence on a behavioral task of impulsivity in healthy subjects. J Stud Alcohol 63: 83-90.

Robbins TW (2002). The 5-choice serial reaction time task: behavioural pharmacology and functional neurochemistry. Psychopharmacology (Berl) 163: 362-380.

Sanchez-Roige S, Pena-Oliver Y, Stephens DN (2012). Measuring impulsivity in mice: the five-choice serial reaction time task. Psychopharmacology (Berl) 219: 253-270.

Saunders JB, Aasland OG, Babor TF, de la Fuente JR, Grant M (1993). Development of the Alcohol Use Disorders Identification Test (AUDIT): WHO Collaborative Project on Early Detection of Persons with Harmful Alcohol Consumption-II. Addiction 88: 791-804.

Scaife JC, Duka T (2009). Behavioural measures of frontal lobe function in a population of young social drinkers with binge drinking pattern. Pharmacol Biochem Behav 93: 354-362.
Stephens DN, Duka T (2008). Review. Cognitive and emotional consequences of binge drinking: role of amygdala and prefrontal cortex. Philos Trans R Soc Lond B Biol Sci 363: 3169-3179.

Townshend JM, Duka T (2002). Patterns of alcohol drinking in a population of young social drinkers: a comparison of questionnaire and diary measures. Alcohol Alcohol 37: 187-192.

Townshend JM, Duka T (2005). Binge drinking, cognitive performance and mood in a population of young social drinkers. Alcohol Clin Exp Res 29: 317-325.

Vonmoos M, Hulka LM, Preller KH, Jenni D, Schulz C, Baumgartner MR et al (2013). Differences in self-reported and behavioral measures of impulsivity in recreational and dependent cocaine users. Drug Alcohol Depend 133: 61-70.

Voon V, Irvine MA, Derbyshire K, Worbe Y, Lange I, Abbott S et al (2014). Measuring 'waiting' impulsivity in substance addictions and binge eating disorder in a novel analogue of rodent serial reaction time task. Biol Psychiatry 75: 148-155.

Walker SE, Pena-Oliver Y, Stephens DN (2011). Learning not to be impulsive: disruption by experience of alcohol withdrawal. Psychopharmacology (Berl) 217: 433-442.

Worbe Y, Irvine M, Lange I, Kundu P, Howell NA, Harrison NA et al (2013). Neuronal correlates of risk-seeking attitudes to anticipated losses in binge drinkers. Biol Psychiatry. http:// dx.doi.org/10.1016/j.biopsych.2013.11.028.

Worbe Y, Savulich G, Voon V, Fernandez-Egea E, Robbins TW (2014). Serotonin depletion induces 'waiting impulsivity' on the human four-choice serial reaction time task: cross-species translational significance. Neuropsychopharmacology 39: 1519-1526.

Supplementary Information accompanies the paper on the Neuropsychopharmacology website (http://www.nature.com/npp) 